\title{
An approximation of balanced score in neutrosophic graphs with weak edge weights
}

\author{
V. Srisarkun ${ }^{1}$, C. Jittawiriyanukoon ${ }^{2}$ \\ ${ }^{1}$ Martin de Tours School of Management and Economics, Assumption University, Thailand \\ ${ }^{2}$ Graduate School of Business and Advanced Technology Management, Assumption University, Thailand
}

\begin{tabular}{l}
\hline \hline Article Info \\
\hline Article history: \\
Received Aug 22, 2020 \\
Revised Apr 21, 2021 \\
Accepted May 19, 2021 \\
\hline
\end{tabular}

Keywords:

Approximation method

Balanced score

Logistic networks

Minimum spanning tree

Undirected neutrosophic graph

\begin{abstract}
Neutrosophic concept is known undirected graph theory to involve with complex logistic networks, not clearly given and unpredictable real life situations, where fuzzy logic malfunctions to model. The transportation objective is to ship all logistic nodes in the network. The logistic network mostly experiences in stable condition, but for some edges found to be volatile. The weight of these erratic edges may vary at random (bridgelifting/bascule, ad hoc accident on road, traffic condition) In this article, we propose an approximation algorithm for solving minimum spanning tree (MST) of an undirected neutrosophic graphs (UNG), in which the edge weights represent neutrosophic values. The approximation upon the balanced score calculation is introduced for all known configurations in alternative MST. As the result, we further compute decisive threshold value for the weak weights amid minimum cost pre-computation. If the threshold triggers then the proper MST can direct the decision and avoid post-computation. The proposed algorithm is also related to other existing approaches and a numerical analysis is presented.
\end{abstract}

This is an open access article under the CC BY-SA license.

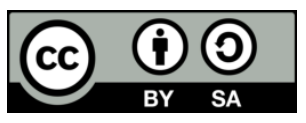

\section{Corresponding Author:}

Chanintorn Jittawiriyanukoon

Graduate School of Business and Advanced Technology Management

Assumption University

Bangkok, 10240, Thailand

Email: pct2526@yahoo.com

\section{INTRODUCTION}

Neutrosophy [1] is a new chapter of science that focuses on the scope of neutralities such as the origin, philosophy and nature. It differs from fuzzy logic [2] which studies the theory of truth degree. Some scholars have announced intuitionistic fuzzy set [3] by introducing the notion of false degree to the set. Later on neutrosophic concept has been firstly brought up by tallying the idea of indeterminate degree to the intuitionistic fuzzy set. Somehow a neutrosophic graph reflects generalities of intuitionistic fuzzy graph but also includes some faults in the definition as explained in [4]. They use some examples to prove that the complement of a neutrosophic graph does not always result a neutrosophic graph, but a graph developed from binary operations such as union, composition, join, is guaranteed to be a new neutrosophic graph. The neutrosophic graph extends the concept of conventional fuzzy set and intuitionistic fuzzy set by adding three independent functions, namely a truth membership (T), an indeterminate membership (I) and a false membership (F). The weights of individual $t, i$ and $f$ are falling within the interval range zero to one for the sake of associating them in real-world complications effectively [5]. The model is helpful as it represents uncertain real-life information such as inconsistency, incompletion or indetermination. It is applicable in multiple fields of engineering and computer science. In general, there are various algorithms [6] for solving 
minimum spanning tree (MST) in graph theory. Currently, MST problems have been elevated by many logistic cases as weights of the edge are not easily predictable, not pre-clarified and yet uncertain. Unless it is beyond our knowledge, there is no investigation in the computer field to engage with the MST problems with vulnerable edge in single valued neutrosophic environment. A graph in the MST has been designed to deal with edges which characterize dissimilarities between two nodes [7]. An estimated heuristic algorithm to optimize the problem over hefty graphs has been presented in [8].

A double-valued neutrosophic graph has been proposed to cluster the data in the MST algorithm and discussed in [9]. An approach to improve the MST algorithm using similarity metrics of computer network problem has been studied in [10]. It is remarked that the triangular fuzzy values differ from a single valued neutrosophic environment.

The focal contribution of this article is to augment the method for solving the minimum cost in the MST algorithm with weak edge weights using an undirected neutrosophic graph. Neutrosophic graphs provide more realistic, accurate, and compatible to real-life logistic applications. The paper will be classified as follows. We introduce the concept of undirected neutrosophic graph and propose a balanced score computation of a single-valued neutrosophic graph. Then we describe the MST algorithm to handle the minimum cost of undirected neutrosophic graph with some weak edges. A numerical example is demonstrated to confirm the proposed function. Finally, a comparative study with existing approaches is performed and subsequently the conclusion is summarized.

\section{UNDIRECTED NEUTROSOPHIC GRAPHS (UNG)}

The degree of vertices is a nontrivial approach to associate the weights of each vertex in a classical graph [11], and we can use the vertex degree for the analysis. The theory of total degree and irregularity in a fuzzy graph has been presented in [12]. A number of properties on the second-degree-vertices and their notation of the fuzzy graph are explained. However, in various real-world applications (i.e. wireless communication networks, logistic networks, and social networks) neutrosophic graphs are much more resilient, cost-effective, accurate, and applicable to model unpredictable problems compared to fuzzy graphs [13].

Moreover, the neutrosophic graph is helpful for achieving an optimization in explicit real-life problems. This knowledge impresses us to eye for numerous types and their inevitable concepts of UNG as reflected most parts in this article. Let $\mathrm{N}$ be an UNG set comprised of three tuples which are the true $\mathrm{T}_{\mathrm{N}}(\mathrm{a})$, indeterminate $\mathrm{I}_{\mathrm{N}}(\mathrm{a})$ and false $\mathrm{F}_{\mathrm{N}}(\mathrm{a})$ members. The single valued UNG is represented by three memberships function $\left(\mathrm{T}_{\mathrm{N}}(\mathrm{a}) \in[0,1], \mathrm{I}_{\mathrm{N}}(\mathrm{a}) \in[0,1], \mathrm{F}_{\mathrm{N}}(\mathrm{a}) \in[0,1]\right)$. Then the degree function of $a$ in $\mathrm{N}$ as shown in (1):

$$
0 \leq \mathrm{T}_{\mathrm{N}}(\mathrm{a})+\mathrm{I}_{\mathrm{N}}(\mathrm{a})+\mathrm{F}_{\mathrm{N}}(\mathrm{a}) \leq 3
$$

\subsection{Minimum spanning tree (MST)}

A spanning tree is a set of undirected graph, in which all nodes are connected with minimum possible number of arcs/edges. It cannot be detached and does not form any cycles. In other words, every undirected and attached graphs always have at least one spanning tree. Thus, the spanning tree has maximum $(=n-1)$ arcs, in which $\mathrm{n}$ is the number of nodes. The MST is principally used to solve a problem of a lowest cost(weight) to cover all nodes in an undirected graph. Certain applications include routing protocol evaluation in computer/communication networks [14], cluster analysis [15] and civil network strategy planning [16]. In weighted graphs, the MST reflects a spanning tree graph which develops minimum cost/weight than other trees on the equivalent graph. In real-life scenarios, the weight can represent computer traffic, distance, expense, congestion, or any subjective values associated to the arcs. MST's well-known methods are Prim's [17] and Kruskal's [18]. Both algorithms' time complexity is $\mathrm{O}\left(a^{*} \log n\right)$, where $a$ and $n$ represent the number of arcs and nodes respectively.

\subsection{Proposed algorithm}

An alternative version of the MST algorithm based on balanced score function is given and dissertated on an UNG with vulnerable edge weights. The MST of an associated neutrosophic graph $\mathrm{N}$ is an acyclic sub-graph which takes on all nodes of graph N. Then the maximum edge includes precisely $a-1$ arcs, in which $a$ represents the total number of nodes of the UNG. In neutrosophic MST, it is the objective to solve that the sum of all decisive arcs/lengths/costs is the lowest. In crumbly situation, the MST algorithm deals with the straight costs linked with the edges of the UNG. However, in real-world environment the arc lengths are fuzzy/vague in natural surroundings. The operation to solve this imprecise weight is to ponder the proposed algorithm for the UNG. In this section, we propose this algorithm to represent the single-valued 
UNG by calculating the balanced score function for any related weights. Let $\mathrm{W}$ (weight) be the membership set of $\left(\mathrm{T}_{\mathrm{w}}, \mathrm{I}_{\mathrm{w}}, \mathrm{F}_{\mathrm{w}}\right)$ then the balanced score function (BS) is approximately computed as shown in (2).

$$
B S(W)=\frac{\left|\left(96+15 T_{w}-40 I_{w}-28 F_{w}\right)\right|}{36}
$$

An UNG with weak edge weights is a single-valued UNG associated with arcs or edges, where there is at least one non-stable edge weight. The arc represents an unknown positive membership $\left(\mathrm{T}_{\mathrm{x}}, \mathrm{I}_{\mathrm{x}} \cdot \mathrm{F}_{\mathrm{x}}\right)$, in which can alter at any time. Other edge weights are stable and their membership figures remain unchanged. The example graph $\mathrm{N}$ illustrated in Figure 1 represents five neutrosophic nodes with five stable arc weights (solid blue arc) and one unstable edge weight (dotted red arc). The weights matrix of this UNG is given by $\left[\left(\mathrm{T}_{\mathrm{x}}, \mathrm{I}_{\mathrm{x}}, \mathrm{F}_{\mathrm{x}}\right),(.2, .5, .8),(.1, .4, .2),(.7, .5, .1),(.6, .3, .9),(.4, .5, .1)\right]$ and corresponding non-zero-neutrosophic adjacency matrix is specified by $[1,1,2,2,2,4][2,4,3,4,5,5]$.

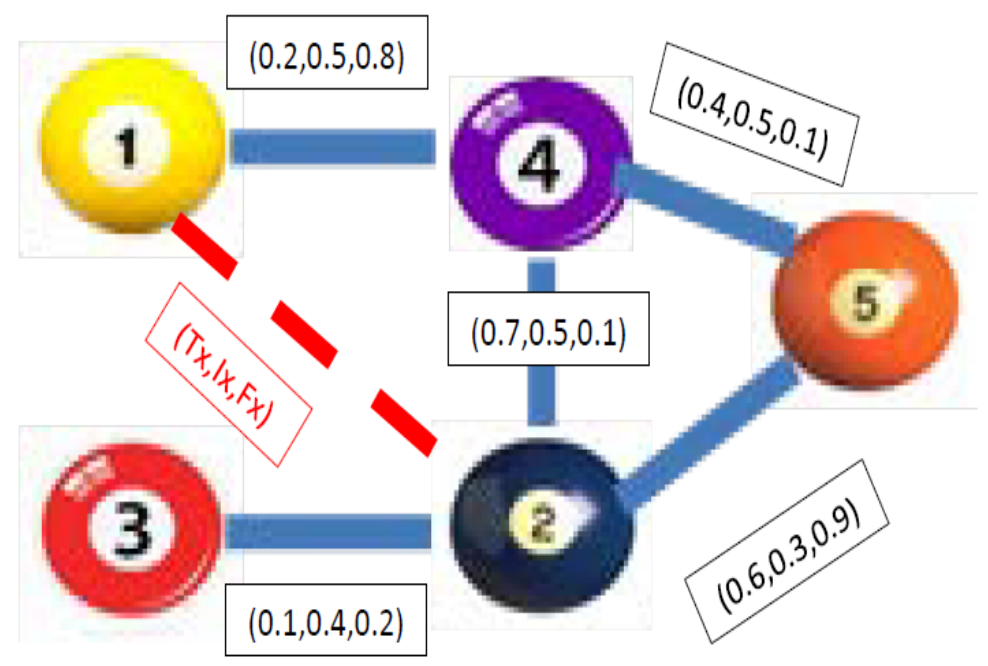

Figure 1. The undirected neutrosophic graph with a vulnerable arc

We can now employ the abovementioned UNG with weak (non-stable) edges. Our proposed algorithm functions in the following five steps. Thus, our proposed algorithm complexity is clearly $\mathrm{O}\left(n^{2}\right)$, where $n$ represents the number of nodes.

Proposed Algorithm:

Input: Non-zero-neutrosophic adjacency matrix and the weight matrix

- $\quad$ Step 1: Convert non-stable $m$ arcs into variable $x_{1}-x_{m}$.

- $\quad$ Iterate until all stable arcs.

- Upon the balanced score calculation $(\mathrm{BS}(\mathrm{W}))$ from (1), substitute these results for all non-zero neutrosophic memberships.

- Step 2: Solve the minimum cost-constrained MST problem (Prim/Kruskal) without the non-stable edges, which will result minimum cost $\mathrm{d}_{\mathrm{A}}$.

- Step 3: Solve the minimum cost-constrained MST problem (Prim/Kruskal) with the compulsory nonstable edges, which will result minimum cost $\mathrm{d}_{\mathrm{B}}+x_{1}+\ldots x_{m}$.

- Step 4: We can identify the decisive values of all $x$.

- $\quad$ Step 5: Terminate.

\section{COMPARATIVE EVALUATION AND ANALYSIS}

We use a practical logistic example of a UNG shown in Figure 2 to demonstrate and compare with other existing approaches upon their score computations. We apply our proposed algorithm's input parameters by giving the weight matrix of the 13-edge-example as $[(.6, .2, .7),(.5, .6, .8),(.8, .2, .8),(.7, .2, .4)$, $(.3, .2, .8),(.2, .2, .3),(.5, .5, .1),(.3, .8, .8),(\mathrm{Tx}, \mathrm{Ix}, \mathrm{Fx}),(.1, .2, .3),(.1, .6, .8),(.6, .2, .7),(.2, .2, .2)]$ and relative nonzero-adjacency matrix is detailed by $[1,1,1,2,2,3,3,3,3,4,5,6,7][2,3,4,3,5,4,5,6,7,6,7,8,8]$. 


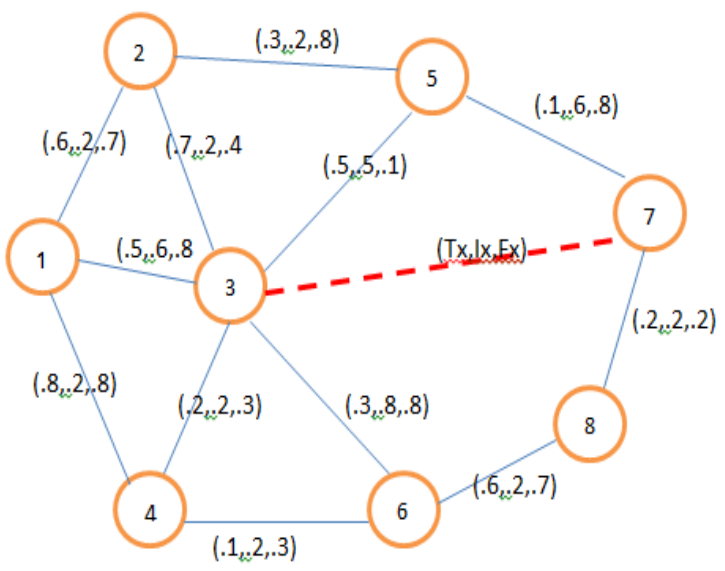

Figure 2. The UNG logistic example

Upon step 1 computation by employing the ten times of the proposed $\mathrm{BS}(\mathrm{W})$, the weight result is $[2.5,8.1,2.4,0.25,4.5,1,1.5,11.1, x, 1.4,9.8,2.5,0.2]$ and depicted in Figure 3. Upon step 2, the result is demonstrated in Figure 4. Upon step 3, the result is listed in Figure 5.

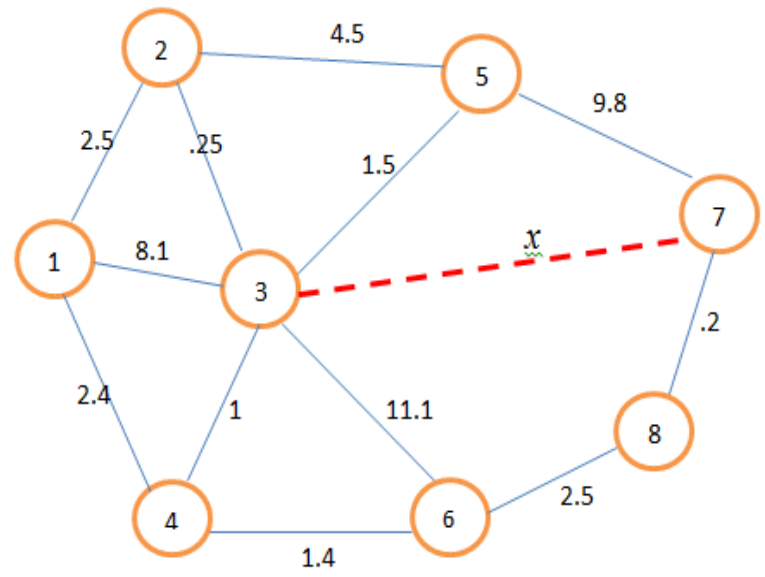

Figure 3. The proposed algorithm upon BS(W) consideration

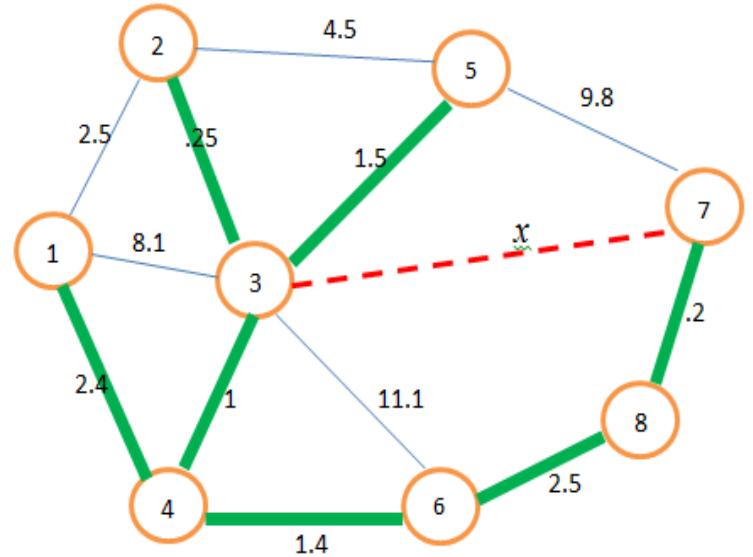

Figure 4. The alternative MST result $\left(\mathrm{d}_{\mathrm{A}}=9.25\right)$ 


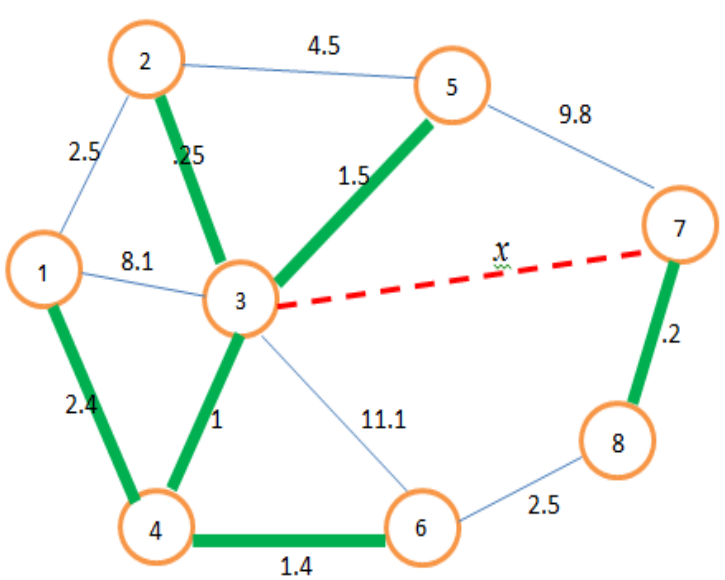

Figure 5. The alternative MST result with weak edge $\left(\mathrm{d}_{\mathrm{B}}=6.75+x\right)$

In the above example, upon step 4 computation we conclude that the crucial value (x) is 2.5 as summarized by step 2 and 3. To rank the UNG membership sets, we perform comparative studies by using a definition of score value function and the relation order between any two nodes presented in [19] and [20]. Nancy and Garg [19] use the neutrosophic membership $\left(T_{x}, I_{x}, F_{x}\right)$ set to generalize the model of intuitionistic and crispy fuzzy sets. They propose the score function for adjusting the uncertain degree between the truth, indeterminate and false degrees. Finally, they demonstrated that their proposed score computation is practical and efficient. Mullai, Broumi, and Stephan [20] use a traditional MST and neutrosophic set to solve the shortest path in a transportation network as the arcs are indeterminate and incomplete. They define a different score function through numerical cases. Thus we take these two score functions into the process of our proposed five-step-algorithm. We employ the above 13-edge logistic network with the weight matrix $[(.6, .2, .7),(.5, .6, .8),(.8, .2, .8),(.7, .2, .4),(.3, .2, .8),(.2, .2, .3),(.5, .5, .1),(.3, .8, .8),(\mathrm{Tx}, \mathrm{Ix}, \mathrm{Fx}),(.1, .2, .3),(.1, .6, .8)$, $(.6, .2, .7),(.2, .2, .2)]$ to evaluate score functions. Weight matrix results are revealed in Table 1 .

Table 1. Score value computation and result

\begin{tabular}{cc}
\hline Score Function & $\mathrm{BS}(\mathrm{W})$ result \\
\hline Proposed algorithm & {$[2.5,8.1,2.4,0.25,4.5,1,1.5,11.1, \mathrm{x}, 1.4,9.8,2.5,0.2]$} \\
Nancy and Garg's algorithm & {$[2.25,7.7,2.1,0.5,3.6,0.65,1,9, \mathrm{n} / \mathrm{a}, 0.5,6.3,2.2,0.3]$} \\
Mullai's algorithm & {$[\mathrm{n} / \mathrm{a}, \mathrm{n} / \mathrm{a}, \mathrm{n} / \mathrm{a}, \mathrm{n} / \mathrm{a}, \mathrm{n} / \mathrm{a}, \mathrm{n} / \mathrm{a}, \mathrm{n} / \mathrm{a}, \mathrm{n} / \mathrm{a}, \mathrm{n} / \mathrm{a}, \mathrm{n} / \mathrm{a}, \mathrm{n} / \mathrm{a}, \mathrm{n} / \mathrm{a}, \mathrm{n} / \mathrm{a}]$} \\
\hline
\end{tabular}

In the table, upon the score function introduced by Nancy's algorithm we can have similar result compared to the proposed algorithm. However, Nancy's algorithm is not applicable to cases such as inconsistency or uncertainty (vulnerable edges). It is apparent that Mullai's algorithm is not capable of obtaining the score value for this example as it focuses particularly on bipolar neutrosophic set. All score values are negative and do not represent real-life edge weights (always positive). The proposed one deals with an alternative MST problem under vulnerable neutrosophic scenarios. It is humble and effective for realworld environment upon the employment of numerical examples, especially in both fuzzy model [21], [22] and artificial intelligence model [23]. Not to mention, the proposed algorithm can help solve a decisionmaking problem on machine [24] and deep learning [25] applications.

\section{CONCLUSION}

The theory of UNG is employed as the outline in fuzzy model and inconsistent environment. There are three memberships of neutrosophic set $\left(\mathrm{T}_{\mathrm{x}}, \mathrm{I}_{\mathrm{x}}, \mathrm{F}_{\mathrm{x}}\right)$. In this article, we propose the new and simple tool in vulnerable edge weight model for solving such a real-life problem. In future, we would investigate other memberships of the artificial intelligence model, e. g. a threshold for training functions, criteria for deep learning and the like. In near future, this research will be extended to the study of setting a threshold for machine and deep learning applications in the artificial intelligence. Furthermore, directed neutrosophic environment is now undergoing. Also some other formulae to compute balanced score function are future scope for our research problem in order to point out the merit of the imminent study. 


\section{REFERENCES}

[1] S. Topal, A. Çevik and F. Smarandache, "A New Group Decision Making Method With Distributed Indeterminacy Form Under Neutrosophic Environment: An Introduction to Neutrosophic Social Choice Theory," in IEEE Access, vol. 8, pp. 42000-42009, 2020, doi: 10.1109/ACCESS.2020.2976872

[2] S. Al-Azzam and A. Sharieh, "A data estimation for failing nodes using fuzzy logic with integrated microcontroller in wireless sensor networks," International Journal of Electrical and Computer Engineering (IJECE), vol. 10, no. 4, pp. 3623-3634, 2020, doi: 10.11591/ijece.v10i4.pp3623-3634.

[3] X. He and Y. Wu, "Global Research Trends of Intuitionistic Fuzzy Set: A Bibliometric Analysis," Journal of Intelligent Systems, vol. 28, no. 4, pp. 621-631, 2019, doi: 10.1515/jisys-2017-0240.

[4] S. Broumi, M. Talea, A. Basali, and F. Smarandache, "Single Valued Neutrosophic Graphs," Journal of New Theory, no. 10, pp. 86-101, 2016, doi: 10.5281/zenodo.50940.

[5] R. Sahin, "An approach to neutrosophic graph theory with applications," Soft Computing, vol. 23, no. 1, pp. 569-581, 2019, doi: 10.1007/s00500-017-2875-1.

[6] N. R. Latha and G. R. Prasad, "Memory and I/O optimized rectilinear Steinerminimum tree routing for VLSI," International Journal of Electrical and Computer Engineering (IJECE), vol. 10, no. 3, pp. 2959-2968, 2020, doi: 10.11591/ijece.v10i3.pp2959-2968.

[7] J. Ye, "Single valued neutrosophic minimum spanning tree and its clustering method," Journal of Intelligent System, vol. 23, no. 3, pp. 311-324, 2014, doi: 10.1515/jisys-2013-0075.

[8] Y. Yang, Z. Li, X. Wang and Q. Hu, "Finding the Shortest Path with Vertex Constraint over Large Graphs," Complexity, vol. 2019, no. 11, pp. 1-13, 2019, doi: 10.1155/2019/8728245.

[9] I. Kandasamy, "Double-Valued Neutrosophic Sets, their Minimum Spanning Trees, and Clustering Algorithm," Journal of Intelligent Systems, vol. 27, no. 2, pp. 163-182, 2018, doi: 10.1515/jisys-2016-0088.

[10] K. Mandal and K. Basu, "Improved similarity measure in neutrosophic environment and its application in finding minimum spanning tree," Journal of Intelligent and Fuzzy System, vol. 31, pp. 1721-1730, 2016, doi: 10.3233/JIFS152082.

[11] T. N. Viet and L. H. Dung, "Extended network and algorithm finding maximal flows," International Journal of Electrical and Computer Engineering (IJECE), vol. 10, no. 2, pp. 1632-1640, 2020, doi: 10.11591/ijece.v10i2.pp1632-1640.

[12] N. R. S. Meheswari and V. Jeyapratha, "Neighbourly Pseudo Irregular Fuzzy Graphs," International Journal of Mathematical Combinatorics, vol. 4, pp. 45-52, 2018, doi: 10.5281/zenodo.3228721.

[13] L. Huang, Y. Li, K. K. Krishnan, D. Koley, and A. Dey, "A Study of Regular and Irregular Neutrosophic Graphs with Real Life Applications,” Mathematics, vol. 7, no. 551, pp. 1-20, 2019, doi: 10.3390/math7060551.

[14] S. Laqtib, K. El Yassini, and M. L. Hasnaoui, "A technical review and comparative analysis of machine learning techniques for intrusion detection systems in MANET," International Journal of Electrical and Computer Engineering (IJECE), vol. 10, no. 3, pp. 2701-2709, 2020, doi: 10.11591/ijece.v10i3.pp2701-2709.

[15] K. V. Rajkumar, A. Yesubabu, and K. Subrahmanyam, "Fuzzy clustering and fuzzy c-means partition cluster analysis and validation studies on a subset of citescore dataset," International Journal of Electrical and Computer Engineering (IJECE), vol. 9, no. 4, pp. 2760-2770, 2019, doi: 10.11591/ijece.v9i4.pp2760-2770.

[16] T. Bhandarkar, K. Vardaan, N. Satish, S. Sridhar, R. Sivakumar, and S. Ghosh, "Earthquake trend prediction using long short-term memory RNN," International Journal of Electrical and Computer Engineering (IJECE), vol. 9, no. 2, pp. 1304-1312, 2019, doi: 10.11591/ijece.v9i2.pp1304-1312.

[17] Y. Li, Y. Zhao, and Y. Zhang, "A spanning tree construction algorithm for industrial wireless sensor networks based on quantum artificial bee colony," EURASIP Journal on Wireless Communications and Networking, vol. 2019, no. 1, pp. 1-12, 2019, doi: 10.1186/s13638-019-1496-z.

[18] T. Zhao, Y. Yang, and E. Wang, "Minimizing the average arriving distance in carpooling," International Journal of Distributed Sensor Networks, vol. 16, no. 1, pp. 1-16, 2020, doi: 10.1177/1550147719899369.

[19] Nancy and H. Garg, "An improved score function for ranking neutrosophic sets and its application to decision making process," International Journal for Uncertainty Quantification, vol. 6, no. 5, pp. 377-385, 2016, doi: 10.1615/Int.J.UncertaintyQuantification.2016018441.

[20] M. Mullai, S. Broumi, and A. Stephen, "Shortest path problem by minimal spanning tree algorithm using bipolar neutrosophic numbers," International Journal of Mathematic Trends and Technology, vol. 46, no. 2, pp. 80-87, 2017, doi: 10.14445/22315373/IJMTT-V46P514.

[21] R. Jafari and W. Yu, "Fuzzy Modeling for Uncertainty Nonlinear Systems with Fuzzy Equations," Mathematical Problems in Engineering, vol. 2017, no. 17-20, pp. 1-10, 2017, doi: 10.1155/2017/8594738.

[22] F. H. Fernandez, E. Kerre, and B. M. Jimenez., "A global fuzzy model for non linear systems using interval valued fuzzy sets," Revista Ingeniería Electrónica, Automática y Comunicaciones, vol. 37, no. 3, pp. 50-57, 2016, doi: 10.1234/RIELAC.V37I3.414.

[23] Hao Zhu, "Big Data and Artificial Intelligence Modeling for Drug Discovery," Annual Review of Pharmacology and Toxicology, vol. 60, pp. 573-589, 2020, doi: 10.1146/annurev-pharmtox-010919-023324.

[24] C. Reda et al., "Machine learning applications in drug development," Computational and Structural Biotechnology Journal, vol. 18, pp. 241-252, 2020, doi: 10.1016/j.csbj.2019.12.006.

[25] N. M. Elaraby, M. Elmogy, and S. Barakat, "Deep Learning: Effective Tool for Big Data Analytics," International Journal of Computer Science Engineering, vol. 5, no. 5, pp. 254-262, 2016. 